03.1

\title{
Влияние динамического напора и молекулярного веса газа на смешение при инжекции струй в поперечный сверхзвуковой поток
}

\author{
(C) Н.Н. Федорова, М.А. Гольдфельд \\ Институт теоретической и прикладной механики им. С.А. Христиановича СО РАН, \\ Новосибирск, Россия \\ E-mail: nfed@itam.nsc.ru
}

Поступило в Редакцию 21 августа 2020 г.

В окончательной редакции 14 сентября 2020 г.

Принято к публикации 16 сентября 2020 г.

Представлены результаты расчетного исследования сверхзвуковых турбулентных течений в канале с уступом при встречной инжекции струй аргона и водорода. Расчеты выполнены при числе Маха 4 на входе в канал для близких к полетным условий, которые были реализованы в опытах в импульсной аэродинамической установке. Проведено сравнение полей течения для коэффициентов динамического напора струй в диапазоне 1-6. Показано, что степень смешения повышается с ростом динамического напора и с увеличением молекулярного веса инжектируемого газа.

Ключевые слова: сверхзвуковой поток, инжекция, динамический напор, смешение.

DOI: 10.21883/PJTF.2021.02.50536.18525

Инжекция струй в сверхзвуковой поток имеет многочисленные инженерные приложения $[1,2]$. В рамках различных программ исследуется применение инжекции газов для управления течением, охлаждения, подачи топлива и др. В последнее время все большее внимание уделяется изучению взаимодействия струй с поперечным сверхзвуковым потоком для понимания структуры потока, глубины проникновения и возможности реализации эффективного смешения при высоких скоростях потока [3-5]. Большинство исследований посвящено инжекции струй в свободный поток при числах Маха $\mathrm{M}=1.5-2.5[1,6]$. Для диапазона скоростей $\mathrm{M}>3$ известны лишь отдельные исследования $[7,8]$. Процесс смешения струй с поперечным основным потоком зависит от конфигурации инжектора, угла подачи, количества инжекторов, химического состава и молекулярной массы впрыскиваемого газа и т. д. [6]. Известно, что нормальная струя может обеспечить лучшее проникновение в ядро потока, чем наклонная $[9,10]$. Процессы смешения и их эффективность существенно зависят от параметров основного потока и молекулярного веса инжектируемого газа, что делает необходимым дальнейшее изучение смешения в таких условиях.

Цель настоящей работы состоит в комплексном исследовании смешения струй водорода и аргона со сверхзвуковым потоком при числе Маха 4 на входе в канал модельной камеры сгорания, соответствующим полетным числам Маха $\mathrm{M}>8$.

Расчеты выполнены для условий экспериментов, проведенных в импульсной установке $[11,12]$ в режиме присоединенного трубопровода. Экспериментальный канал состоит из сверхзвукового сопла, изолятора, инжекторной секции с двумя уступами высотой $25 \mathrm{~mm}$, за которой находятся секция постоянного сечения вы- сотой $h=100 \mathrm{~mm}$ и расширяющаяся секция с углом раскрытия $12^{\circ}$. Продольное сечение канала показано на рис. 1, $a$. Ширина канала в поперечном направлении составляет $100 \mathrm{~mm}$. На верхней и нижней стенках на расстоянии $12.5 \mathrm{~mm}$ перед уступом находятся круглые отверстия диаметром $2.8 \mathrm{~mm}$ для инжекции водорода и аргона. В качестве основного газа использовался азот для того, чтобы предотвратить воспламенение водорода при высоких температурах воздуха. В экспериментах измерялись распределение статического давления на стенках модели, давление за прямым скачком (данные трубки Пито) на входе и выходе канала и расходы первичного и впрыскиваемого газа. Модель испытывалась при следующих условиях на входе в канал: число Маха $\mathrm{M}_{\infty}=4$, полная температура $T_{0 \infty}=1700 \mathrm{~K}$, полное давление в канале $P_{0 \infty}=5.5-6.5 \mathrm{MPa}$. Газ инжектировался под углом $90^{\circ}$ к основному потоку. Коэффициент динамического напора струи $J=\left(\rho U^{2}\right)_{j e t} /\left(\rho U^{2}\right)_{\infty}=\left(\gamma p \mathrm{M}^{2}\right)_{j e t} /\left(\gamma p \mathrm{M}^{2}\right)_{\infty}$ (где $\rho-$ плотность, $U-$ скорость, $\gamma-$ показатель адиабаты, $p$ - статическое давление), равный отношению динамического напора струи (jet) к динамическому напору в набегающем потоке $(\infty)$, изменялся в диапазоне от 0.7 до 6. Более подробно экспериментальная установка и методы измерений описаны в [12].

Для численного решения использован программный комплекс ANSYS Fluent. Расчеты проведены на основе стационарных осредненных по Рейнольдсу уравнений Навье-Стокса для многокомпонентной смеси, дополненных $k-\omega$ SST-моделью турбулентности. Использовался решатель, основанный на плотности. Для аппроксимации конвективных членов использована схема AUSM второго порядка. 

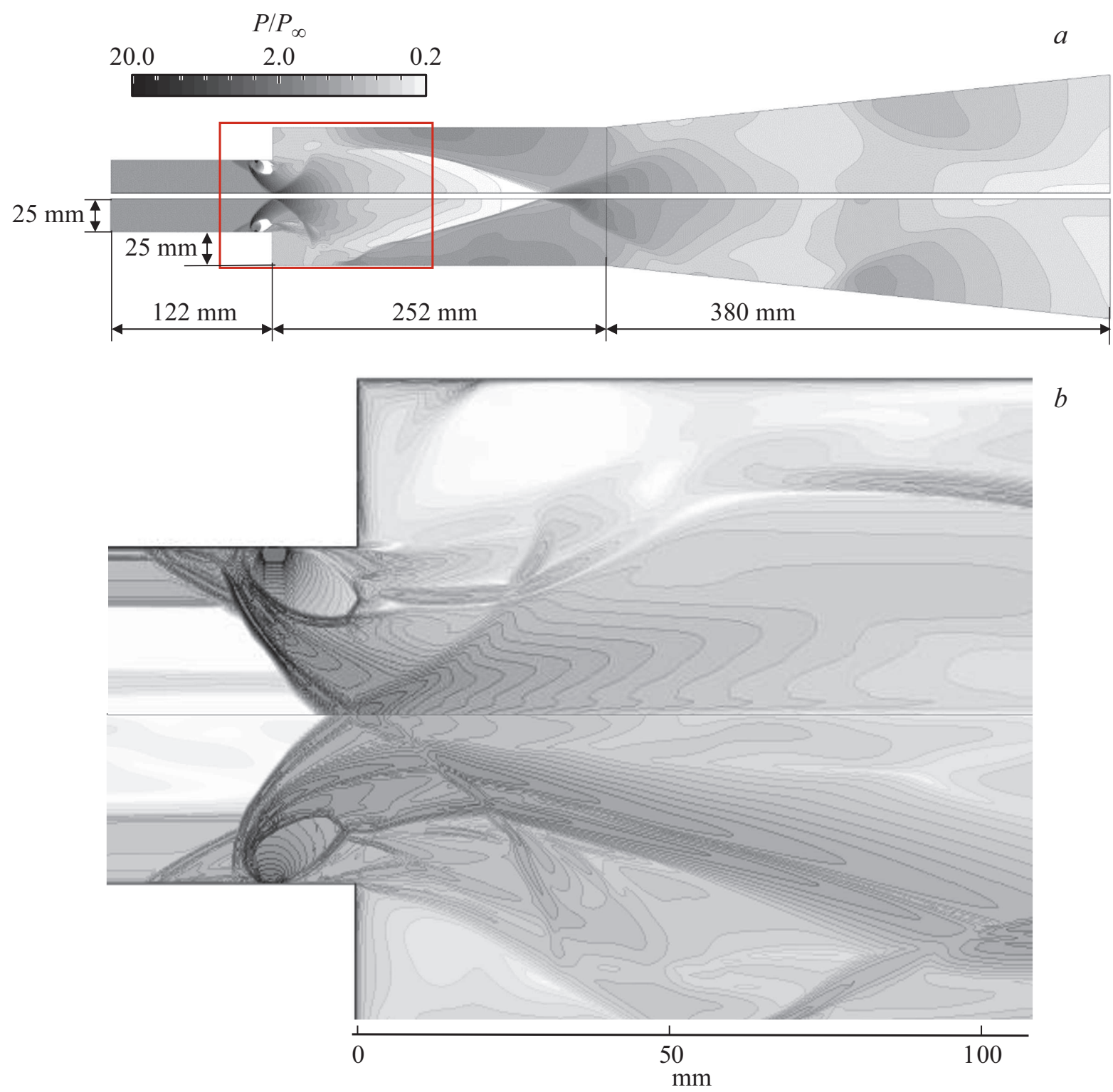

Рис. 1. Схема расчетной области и распределение статического давления в центральном продольном сечении (a) и шлиренкартины течения вблизи уступа $(b)$ для инжекции струй водорода (сверху) и аргона (снизу), $J=6$.

Трехмерная расчетная область включает инжектор, секцию постоянного сечения и расширяющуюся секцию с учетом симметрии в вертикальном и горизонтальном направлениях. Начало расчетной системы координат находится на плоскости симметрии во внутреннем угле уступа. На нижней и задней стенках заданы условия прилипания и „холодной“ стенки $T_{w}=300 \mathrm{~K}$, на фронтальной и верхней границах расчетной области - условия симметрии. На входе задано статическое давление и профили числа Маха с учетом пограничного слоя толщиной $11 \mathrm{~mm}$. Поскольку поток сверхзвуковой, заданные на выходе значения статического давления оказывают влияние на решение в дозвуковой области вблизи стенки в нескольких ячейках вверх по потоку от выхода из канала.
Для расчетов использовалась многоблочная структурированная гексагональная сетка со сгущением к стенкам и зоне инжекции. Для каждого газа при одном значении $J$ было проведено исследование сеточной сходимости на последовательности сеток, содержащих 5, 6.5 и 10 млн ячеек, которые получены путем адаптации начальной сетки по градиенту плотности и величине $y^{+}$, характеризующей размер сетки вблизи стенок. При расчете турбулентных отрывных течений эта величина должна быть порядка единицы, чтобы разрешить ламинарный подслой. Нужные значения были достигнуты с помощью автоматической адаптации Fluent. Исходная сетка измельчалась вблизи стенки до получения сеточно-независимого решения. Сравнение показало совпадение результатов по распределению дав- 
ления на стенках, полученных на средней и мелкой сетках. Представленные далее результаты получены на средней сетке со значениями $y^{+}$на стенках, не превышающими 5. Подробное описание метода и технологии расчета можно найти в работах $[13,14]$, в которых проведено тестирование численного алгоритма и представлены результаты численных исследований сверхзвуковых течений в каналах при больших числах Маха.

На рис. 1, а представлено сравнение полей нормированного статического давления в центральном продольном сечении канала для случая инжекции струй водорода (сверху) и аргона (снизу) при $J=6$. Рамкой выделена область, для которой на рис. $1, b$ проведено сравнение расчетных картин течения для случаев инжекции водородной и аргоновой струй. Темные тона и сгущение изолиний на рис. $1, b$ соответствуют высоким, а светлые тона и отсутствие изолиний - низким градиентам плотности.

Расчет позволяет детально проанализировать картину течения как в области инжекции струи, так и по всей длине канала. На рис. $1, a, b$ можно видеть $\lambda$-конфигурацию, образованную формирующейся перед струей головной ударной волной и отрывным скачком, а также бочкообразную структуру внутри струи, замыкающуюся диском Маха. Головной скачок приходит на верхнюю границу расчетной области (плоскость симметрии) и отражается от нее. При прохождении через струю водорода, имеющую низкую плотность, скачок отражается от нее волной разрежения и практически исчезает (рис. $1, b$ ). Струя аргона имеет существенно более высокую плотность по сравнению с внешним потоком, поэтому головной скачок проникает ближе к стенке и исчезает на границе дозвуковой отрывной зоны за уступом. За отраженным головным скачком поток разворачивается к стенке, в результате образуется веер волн разрежения, интенсивность которого увеличивается за счет волн, формирующихся при отражении головного скачка от зон низкой плотности. Отрывная область замыкается хвостовым скачком, который в случае водородной струи формируется ниже по течению и ближе к центру канала и имеет более пологий профиль. Для аргоновой струи скачок формируется вблизи стенки и искривляется при прохождении через струю, имеющую высокую плотность.

Рис. $1, b$ показывает, что структуры течения в области инжекции струй водорода и аргона идентичны, и высота проникновения струй примерно одинаковая, что можно объяснить одинаковым значением $J$. Однако струя водорода порождает „бочку“ большего по сравнению с аргоном размера. Границы струи аргона очень четкие, что хорошо видно по сгущению изолиний градиента плотности, которое выделяют области с массовой концентрацией аргона выше 0.6. Плотность струи водорода ниже, чем плотность первичного газа, поэтому ее границы можно восстановить только по излому изолиний градиента плотности.



Рис. 2. Распределение нормализованного статического давления на стенке канала за уступом при $J=0$ и при инжекции водорода и аргона для $J=6$. Расчет - данные настоящей работы, эксперимент - данные [12].

Максимальное давление достигается в области за головным скачком, минимальное - внутри „бочки“, где струя резко расширяется и газ ускоряется, и в центре канала, куда приходят волны разрежения с противоположных стенок. Система волн сжатия/разрежения распространяется по каналу, отражаясь от стенок и формируя характерный пилообразный профиль давления (рис. 2). Сравнение распределений давления на стенке показывает, что струя приводит к повышению донного давления и сдвигу волновой конфигурации вверх по потоку, и этот эффект усиливается с ростом $J$. Для струи аргона за уступом получено немонотонное распределение давления с двойным максимумом. Первый максимум возникает в зоне формирования хвостового скачка, а второй обусловлен действием головного скачка, отраженного от боковой стенки канала. Для водородных струй распределение давления более гладкое. Максимум давления в расширяющейся части канала $(x \approx 0.3)$ обусловлен действием хвостового скачка с противоположной стенки. На расчетной кривой появляется дополнительный локальный максимум давления при $x \approx 0.45 \mathrm{~m}$, который образуется в месте падения головного скачка с противоположной стенки, отраженного от боковой стенки канала. Сравнение расчетных и экспериментальных данных [12] показывает их качественное соответствие при изменении $J$.

Для количественной оценки уровня давления во всем исследованном диапазоне $J$ в таблице приведены расчетные и экспериментальные значения локальных максимумов давления за хвостовым скачком $\left(\max _{1}\right)$, за отраженным хвостовым скачком $\left(\max _{2}\right)$ и минимума в донной области ( $\mathrm{min})$. Можно видеть, что различие расчетных и экспериментальных данных не превышает $20 \%$. Наибольшее различие наблюдается в донной области, что может быть связано с погрешностями измерения низкого давления за уступом или недостаточным количеством приемников давления. 
Максимальные и минимальные значения нормализованного статического давления на стенках канала и относительная погрешность определения

\begin{tabular}{c|c|c|c|c|c|c|c|c|c|c}
\hline \multirow{2}{*}{ Газ } & \multirow{2}{*}{$J$} & \multicolumn{3}{|c|}{$\max _{1}$} & \multicolumn{3}{c|}{$\max _{2}$} & \multicolumn{3}{c}{$\min$} \\
\cline { 3 - 10 } & & Эксперимент & Расчет & $\begin{array}{c}\text { Относительная } \\
\text { погрешность, \% }\end{array}$ & Эксперимент & Расчет & $\begin{array}{c}\text { Относительная } \\
\text { погрешность, \% }\end{array}$ & Эксперимент & Расчет & $\begin{array}{c}\text { Относительная } \\
\text { погрешность, \% }\end{array}$ \\
\hline- & 0 & 1.03 & 1 & 3 & 0.47 & 0.46 & 2 & 0.135 & 0.136 & 0.7 \\
$\mathrm{Ar}$ & 3 & 1.14 & 1 & 12 & 0.49 & 0.48 & 2 & 0.247 & 0.2 & 19 \\
& 4.5 & 1.11 & 1.05 & 5.4 & 0.512 & 0.593 & 13.7 & 0.287 & 0.302 & 5 \\
$\mathrm{H}_{2}$ & 6 & 1.256 & 1.22 & 2.9 & 0.593 & 0.73 & 19 & 0.385 & 0.342 & 11 \\
& 1.25 & 1.03 & 16 & 0.49 & 0.46 & 6 & 0.24 & 0.19 & 20 \\
& 4.5 & 1.28 & 1.09 & 15 & 0.497 & 0.51 & 2.5 & 0.36 & 0.26 & 28 \\
& 6 & 1.33 & 1.153 & 13 & 0.548 & 0.561 & 2.5 & 0.380 & 0.374 & 1.6
\end{tabular}

Важной характеристикой камеры сгорания является коэффициент потерь полного давления по длине канала. При отсутствии струй коэффициент потерь полного давления, вычисленный как отношение усредненного по площади выходного и входного сечений полного давления, составляет 0.78 . С ростом $J$ коэффициент снижается, минимальные значения при $J=6$ составляют 0.47 для инжекции водорода и 0.27 для аргона.

На рис. 3 представлены результаты расчета массовой концентрации водорода и аргона в вертикальной плоскости симметрии и на нижней стенке канала. Непосредственно за отверстием инжекции максимальная концентрация достигается вблизи плоскости симметрии (ядро потока), но под воздействием головного скачка с противоположной стенки максимум концентрации смещается к стенке канала. Воздействие хвостового скачка вновь приводит к смещению максимума концентрации в ядро потока, но его величина значительно снижается. В вертикальной плоскости основная масса инжектируемого газа находится вблизи ядра потока. Небольшое расширение области смешения в поперечном направлении происходит под воздействием давления за куполообразным головным скачком, однако вблизи боковой стенки концентрация инжектируемого газа остается низкой. Рисунок показывает существенную неоднородность концентраций по длине канала и в поперечном направлении. Можно видеть, что концентрация аргона в плоскости симметрии выше концентрации водорода, и размер области с ненулевыми значениями концентрации аргона у нижней стенки больше, чем в случае водорода.

Для оценки степени смешения вторичного и основного газа в поперечных сечениях вдоль канала вычислены значения осредненного по массе индекса однородности $\gamma_{m}$ :

$$
\bar{\phi}_{m}=\frac{\sum_{i} \phi_{i}\left|\rho_{i} U_{i} A_{i}\right|}{\sum_{i}\left|\rho_{i} U_{i} A_{i}\right|}, \quad \gamma_{m}=1-\frac{\sum_{i}\left(\left|\phi_{i}-\bar{\phi}_{m}\right|\right)\left|\rho_{i} U_{i} A_{i}\right|}{2\left|\bar{\phi}_{m}\right| \sum_{i}\left|\rho_{i} U_{i} A_{i}\right|} .
$$

Здесь $\phi_{i}$ - массовая концентрация вторичного газа в $i$-й расчетной ячейке; $\rho_{i} U_{i}-$ массовый расход через поперечное сечение $i$-й ячейки, $A_{i}$ - площадь сечения, $\bar{\phi}_{m}-$ средняя массовая концентрация вторичного газа по всему сечению. Значения индекса однородности, представленные на рис. 3,c, показывают, что степень смешения газов монотонно растет по длине канала и повышается с ростом $J$. При этом индекс однородности аргона выше индекса однородности водорода при всех исследованных значениях $J$ по всей длине канала за уступом.

На основе анализа результатов, представленных на рис. 3, можно заключить, что одно отверстие инжекции по ширине канала $100 \mathrm{~mm}$ не позволяет получить высокий уровень смешения. Даже при максимальном значении $J$ индекс однородности аргона в конце канала не превышает 0.5 , что связано с наличием областей с низкой концентрацией аргона. Уровень смешения водорода в конце канала при всех $J$ на 40\% ниже соответствующих значений для аргона. Показано, что воздействие хвостового и отраженного от боковой стенки головного скачка уплотнения приводит к образованию зоны с благоприятными для воспламенения значениями массовой концентрации водорода 0.0264-0.0141, соответствующими коэффициентам избытка топлива 0.91-0.48. Результаты численного моделирования смешения водородной струи с инертным газом позволили обосновать возможность воспламенения водородовоздушной смеси в средней части секции постоянного сечения [15], а также применить полученные данные для оценки эффективности смешения газообразных углеводородных топлив (метан $\mathrm{CH}_{4}$, этилен $\mathrm{C}_{2} \mathrm{H}_{4}$, пропан $\mathrm{C}_{3} \mathrm{H}_{8}$ ) с существенно более высоким молекулярным весом.

Таким образом, проведенное численное моделирование взаимодействия струй водорода и аргона с поперечным сверхзвуковым потоком показало, что инжекция газа в поперечный поток сопровождается повышением давления по длине канала с образованием отчетливо выраженных локальных максимумов. Значения максимумов увеличиваются с ростом динамического давления, и на их величину влияет молекулярный вес инжектируемого газа. Увеличение динамического напора подачи газа 

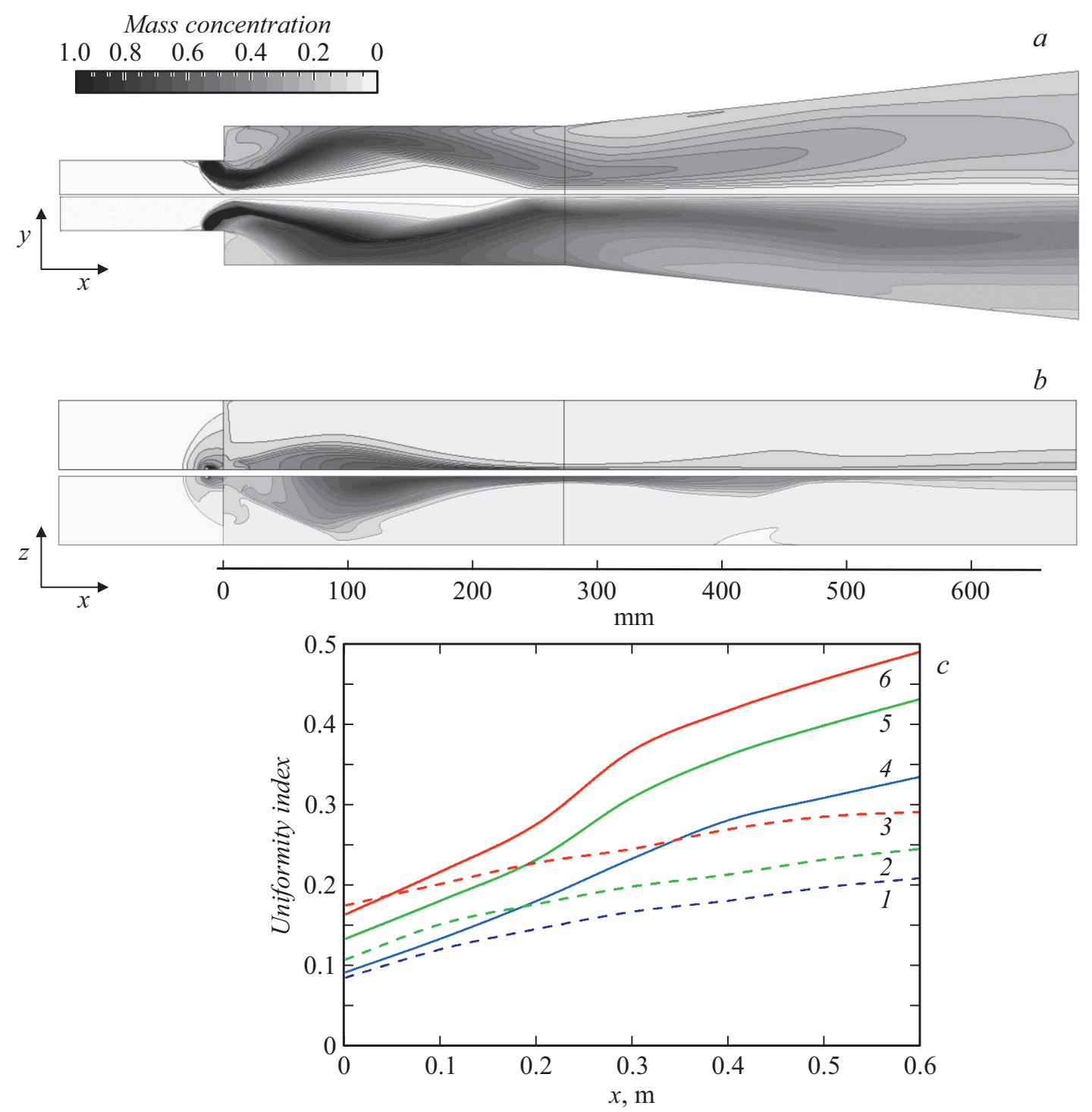

Рис. 3. Расчетные поля массовой концентрации водорода (сверху) и аргона (снизу) для $J=6$ в центральном продольном сечении $(a)$ и на нижней стенке $(b) ; c-$ расчетный индекс однородности водорода $(1-3)$ и аргона $(4-6)$ при $J=3(1,4)$, $4.5(2,5)$ и $6(3,6)$.

повышает степень смешения, при этом потери полного давления также растут. Повышение молекулярного веса газа сопровождается существенной интенсификацией степени смешения и ее повышением вниз по потоку. Для аргона коэффициент потерь полного давления значительно ниже, чем для водорода.

\section{Финансирование работы}

Работа выполнена в рамках Программы фундаментальных научных исследований государственных академий наук на 2013-2020 гг. (проект AАAА-A17117030610139-4) при частичной поддержке Российского фонда фундаментальных исследований (грант № 20-0801158).

\section{Конфликт интересов}

Авторы заявляют, что у них нет конфликта интересов.

\section{Список литературы}

[1] K. Mahesh, Annu. Rev. Fluid Mech., 45, 379 (2013). DOI: 10.1146/annurev-fluid-120710-101115

[2] К.Н. Волков, В.Н. Емельянов, М.С. Яковчук, ЖТФ, 89 (3), 353 (2019). DOI: 10.21883/JTF.2019.03.47168.47-18 [Пер. версия: $10.1134 / \mathrm{S} 1063784219030265]$.

[3] Z. Rana, B. Thornber, D. Drikakis, Phys. Fluids, 23, 046103 (2011). DOI: 10.1063/1.3570692

[4] M. Sun, H. Wang, F. Xiao, In Jet in supersonic crossflow (Springer, Singapore, 2019), p. 27-53. DOI: 10.1007/978-98113-6025-1_2 
[5] C.H. Liang, M.B. Sun, Y. Liu, Y.X. Yang, Acta Astron., 148, 12 (2018). DOI: 10.1016/j.actaastro.2018.04.009

[6] W. Huang, Aerospace Sci. Technol., 50, 183 (2016). DOI: $10.1016 / j$.ast.2016.01.001

[7] J.A. Schetz, L. Maddalena, S.K. Burger, J. Propul. Power, 26, 1102 (2010). DOI: $10.2514 / 1.49355$

[8] A.S. Pudsey, V. Wheatley, R.R. Boyce, J. Propul. Power, 31, 144 (2015). DOI: 10.2514/1.B35298

[9] M. Gamba, M.G. Mungal, J. Fluid Mech., 780, 226 (2015). DOI: $10.1017 / \mathrm{jfm} .2015 .454$

[10] W.O. Landsberg, V. Wheatley, A. Veeraragavan, AIAA J., 54, 3692 (2016). DOI: $10.2514 / 1 . j 054815$

[11] M.A. Goldfeld, A.A. Maslov, A.V. Starov, V.V. Shumskii, M.I. Yaroslavtsev, AIP Conf. Proc., 1770, 030020 (2016). DOI: $10.1063 / 1.4963962$

[12] М.П. Голубев, М.А. Гольдфельд, Письма в ЖТФ, 45 (1), 50 (2019). DOI: 10.21883/PJTF.2019.01.47158.17517 [Пер. версия: 10.1134/S1063785019010073].

[13] Н.Н. Федорова, И.А. Федорченко, А.В. Федоров, ПМТФ, $54(2), 32$ (2013). [Пер. версия: 10.1134/S002189441302003X].

[14] М.А. Гольдфельд, Ю.В. Захарова, А.В. Федоров, Н.Н. Федорова, ФГВ, 54 (6), 3 (2018). [Пер. версия: 10.1134/S0010508218060011].

[15] M.A. Goldfeld, K.Yu. Timofeev, AIP Conf. Proc., 1893, 030147 (2017). DOI: 10.1063/1.5007605 